\title{
Fault-tolerant unfalsified control for PEM fuel cell systems
}

\author{
Fernando D. Bianchi, Carlos Ocampo-Martinez, Senior Member IEEE, Cristian Kunusch, Member IEEE, \\ and Ricardo S. Sánchez-Peña, Senior Member IEEE
}

\begin{abstract}
The article addresses the implementation of a datadriven control strategy in a real test bench based on proton exchange membrane fuel cells (PEMFCs). The proposed control scheme is based on Unfalsified Control (UC), which allows adapting in real-time the control law by evaluating the performance specifications based only on measured input-output data. This approach is especially suitable to deal with non-linearity, model uncertainty and also possible faults that may occur in PEMFCs. The control strategy has been applied to several experimental practical situations in order to evaluate not only the system performance but also different fault scenarios. The experimental results have shown the effectiveness of the proposed approach to regulate the oxygen stoichiometry in real-time operation as well as to maintain a proper system performance under fault situations. Also, a start-up mass-flow controller is added in order to bring the system towards its normal operating conditions.
\end{abstract}

Index Terms-Unfalsified control; PEM fuel cells; oxygen stoichiometry; fault-tolerant control tests.

\section{INTRODUCTION}

$\mathbf{T}$ HE evolution of modern society has been mostly based on the consumption of fossil fuel for electricity generation and the functioning of critical infrastructures such as transport networks. This model is strongly dependent on the constantly decreasing reserves of that type of fuel, which is also related to hazardous problems such as global warming. However, there are several options for electricity generation beyond fossil fuels that could mitigate the dependence modern society has with these scarce and polluting resources. Clean energy sources, and in particular, fuel cells (FCs) as electrochemical devices that generate electrical energy from hydrogen and oxygen, with pure water and heat as byproducts, are regarded as one

F.D. Bianchi is with Catalonia Institute for Energy Research, IREC, Jardins de les Dones de Negre 1, 08930 Sant Adrià de Besòs, Barcelona, Spain. email: fbianchi@irec.cat

C. Ocampo-Martinez and C. Kunusch are with Universitat Politècnica de Catalunya, Institut de Robòtica i Informàtica Industrial (CSIC-UPC), Llorens i Artigas, 4-6, 08028 Barcelona, Spain.

R.S. Sánchez-Peña is with CONICET and Instituto Tecnológico de Buenos Aires (ITBA), Av. Madero 399, (C1106ACD) Buenos Aires, Argentina.

The research of C. Ocampo-Martinez and C. Kunusch has been supported by the project MACPERCON (Ref. 201250E027) of the CSIC.

The research of $\mathrm{C}$. Kunusch has been also supported by the 7th Framework Programme of the EU through the Marie Curie actions (GA: PCIG09-GA2011-293876) and Puma-Mind project (GA: FCH-JU-2011-1-303419), as well as by the CICYT project DPI2011-25649 (MICINN-Spain).

The research of R.S. Sánchez Peña has been supported by CONICET and grant PICT2008-290 from the PRH Program of the Ministry of Science, Technology and Innovation of Argentina.

Color versions of one or more of the figures in this paper are available online.

Manuscript received February 15, 2014 ; revised August 22, 2014. of the most promising technologies due to their potential efficiency, compactness and reliability [1]. Important advances in the design of these devices as well as on their materials allow to consider FCs viable for electricity generation not only at small scale (automotive) but also as technologies embedded in complex arrays of poly-generation such as the so called smart energy grids [2]. In particular, Polymer Electrolyte Membrane FCs (PEMFCs) are a type of FCs especially developed for both portable and stationary applications. Their distinguished features include lower pressure ranges, temperatures from $45^{\circ} \mathrm{C}$ to $95^{\circ} \mathrm{C}$, and a special polymer electrolyte membrane (conducting hydrogen protons) [3].

Despite the notorious advantages of these devices and the widespread availability of hydrogen as a fuel, several technological challenges related to the PEMFC efficiency, lifetime and economical costs are still open as major limitations for their standard implementation in everyday solutions. This fact, together with the recent advances in material sciences and component enhancements, make advanced control techniques appear as complementary strategies in order to reduce costs, improve performance and optimize efficiency, therefore increasing the lifetime of PEMFC-based systems. Hence, reliable control systems may ensure system stability and performance, as well as robustness against uncertainties and exogenous perturbations, all properties of capital importance for PEMFC success. Several research works have addressed the oxygen stoichiometry control to optimize the system conversion efficiency, avoiding performance deterioration together with eventual irreversible damages in the polymeric membranes due to oxygen starvation. These works present the way to achieve the aforementioned control objective by using different techniques: Model Predictive Control (MPC) [4], Sliding-Mode Control [5], Full-state Feedback with Integral Control [6] or LQR/LQG-based control [7], Linear Parameter Varying (LPV) control [8], adaptive control [9], among others.

One important aspect when controlling real systems is concerned with the occurrence of component faults and their influence in the overall system performance. In fact, faults and model/sensor/actuator uncertainty play similar roles, then the conceptual distinction among them represents the difference between active $e^{1}$ and passive ${ }^{2}$ fault-tolerant control (FTC) design approaches [10]. In the framework of FCs and

\footnotetext{
${ }^{1}$ Active FTC strategies aim at adapting the control loop based on the information provided by a fault detection and isolation (FDI) module within the fault-tolerant architecture.

${ }^{2}$ In passive FTC strategies, a single control law is used in both faultless and faulty operation, assuming a certain degree of performance degradation.
} 
assuming an active FTC architecture, several approaches for fault detection and isolation (FDI) have been proposed. Modelbased FDI for PEMFC systems based on consistency relations for the detection and isolation of predefined faults has been proposed in [11], while in [12] a comparison of both modelbased and data-driven fault detection methods for FCs is addressed. The work in [13] proposes a methodology to use the electrical model for FC system diagnosis, while in [14] a fault diagnosis and accommodation system based on fuzzy logic has been developed as an effective complement for a closedloop scheme. Regarding FTC, [15] presents an experimental implementation of an active FTC system for a FC/battery hybrid power-train applied to a city bus, while [16] proposes an MPC scheme for adding fault tolerance capabilities to a two-actuator PEMFC system.

Unfalsified Control (UC) theory was born as an approach for data-driven control, where no prior hypothesis on the plant is used besides the measured data streams [17]. The control law is selected from a predefined set by the performance evaluation based solely on the information provided by the measured input-output data. The controllers that do not achieve the desired performance specifications are discarded (falsified). Instead, one of the remaining (unfalsified) controllers is used until it is falsified by the past measurements and replaced by a new unfalsified controller and so on. This technique has been formally introduced by Safonov and co-workers [18]. UC is a real-time implementation method that may be combined with other model-based design techniques, hence it is not mutually exclusive [19].

At this point, UC emerges as an especially suitable technique to tackle the complex characteristics inherent to FC systems. Non-linear dynamics, inaccessible variables and model uncertainties are natural addresses by UC. Being a datadriven approach, UC is also particularly suited for dealing with unknown disturbances and possible fault occurrences. The application of UC in other systems has been previously reported in the literature and ranges from chemical reactors [20], flight control systems [21], up to micro-aerial vehicles [22], among others. In [23], the implementation of an ellipsoidal UC (EUC) in a dual rotary 4th order motion system is presented, showing the success of the experimentation by ensuring the convergence of the proposed algorithm. By a suitable selection of the controller set and the performance test, EUC is capable of an efficient implementation of UC ideas as a convex optimization problem easily implemented in real-time. From the best of the author's knowledge, UC has never been implemented in the control/supervision of a complex system based on PEMFCs.

The main contribution of this paper is a robust oxygen stoichiometry control design based on UC and its implementation in a laboratory FC system. In particular, an EUC-based closedloop scheme [24] is designed and tested experimentally under several scenarios. The control objectives cover the traditional stoichiometry regulation, disturbance rejection represented by changes in the load profile of the PEMFC, and also the consideration of actual fault events in the components, which may induce performance loss and hazardous operation of the entire system. The proposed approach may be integrated into a multi-level supervisory control scheme, where other system variables might be simultaneously regulated towards the improvement of global objectives such as durability and efficiency of the overall PEMFC system [25]. Experimental results have shown the effectiveness of the proposed approach in fulfilling the control objective (stoichiometry regulation) in real-time system operation. The overall scheme proposed in this paper also includes a start-up mass-flow control strategy, which avoids an abrupt/non-smooth behavior of the system variables when the EUC controller is started with initial conditions far away from the nominal system operation. The scheme proposed in this paper introduces fault tolerance capabilities as in [26] but considering the proposed fault scenarios over the real experiment.

The remainder of the paper is organized as follows. Section II briefly describes the physical system and control objectives as well as the main parts of the experimental test bench. Section III introduces the EUC techniques as well as the necessary modifications in order to implement it in the real case presented here. Section IV collects and explains in detail the experimental results for different practical scenarios, and Section V presents the main conclusions.

\section{System Physical Description}

The system is comprised by a central PEMFC stack and additional/complementary units. In Fig. 1 the scheme of the considered system and the interaction between its different subsystems (FC stack, reactant supply system and humidity management unit) is shown. A brief description of some components, variables and processes is presented as follows. In the system, the control input $u$ corresponds to the compressor voltage denoted $V_{c p}$. The system output $y$ corresponds in turn to the inlet stoichiometry of the PEMFC cathode, namely $\lambda_{O_{2}}$. Moreover, the system is affected by the external disturbance $I_{s t}$, which corresponds to the stack current flowing towards the load.

The main subsystems depicted in Fig. 1 are:

- A $12 \mathrm{~V}$ DC air compressor with an oil-free diaphragm vacuum pump, whose input voltage $V_{c p}$ is the control variable (as established beforehand).

- Hydrogen and oxygen Cellkraft ${ }^{\circledR}$ membrane exchange humidifiers and line heaters, which are used to maintain proper humidity and temperature conditions inside the cell stack $^{3}$.

- A ZBT ${ }^{\circledR} 8$ fuell-cell stack with Nafion $115^{\circledR}$ membrane electrode assemblies with $50 \mathrm{~cm}^{2}$ of active area and $150 \mathrm{~W}$ power.

Moreover, different sensors are incorporated into the system such as an air-mass flowmeter (range 0-15 slpm) at the end of the compressor to measure its flow $\left(W_{c p}\right)$, a current clamp (range $0-3 \mathrm{~A}$ ) and a voltage meter (range $0-15 \mathrm{~V}$ ) to measure the motor stator current $\left(I_{c p}\right)$ and voltage $\left(V_{c p}\right)$, respectively. Besides, temperature sensors are arranged in order to register the different operation conditions. The full description of this

\footnotetext{
${ }^{3}$ Decentralized PID controllers are in charge of ensuring the adequate operation values for these devices, therefore this control design is out of the scope of this paper.
} 


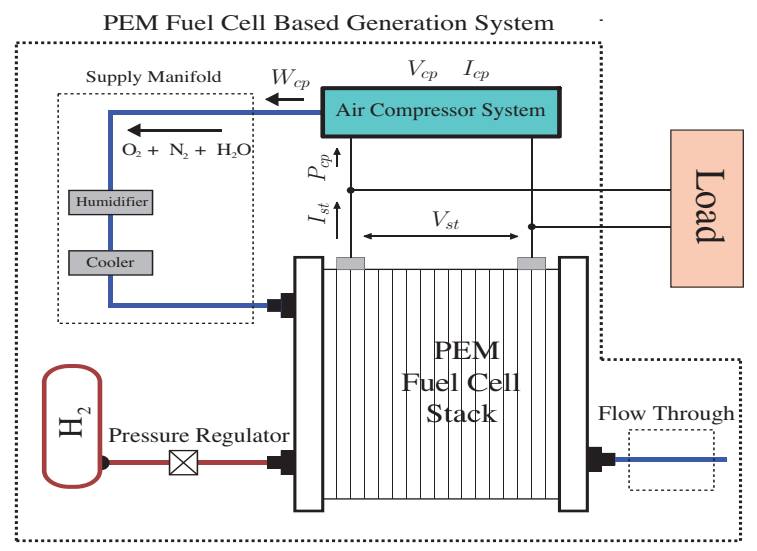

Fig. 1. Schematic diagram of the PEMFC based generation system

system as well as a fully-validated nonlinear dynamic model specially developed for control purposes, are presented and deeply discussed in [3]. Given the complexity of the nonlinear model and the consequent difficulty for designing and implementing online controllers, data-driven control techniques rise as an attractive alternative for real-time operation of such systems, mainly when different experimental scenarios are considered.

In order to maximize the efficiency of the PEMFC system, the regulation of the oxygen mass inflow towards the stack cathode should be achieved. Additionally, oxygen starvation and irreversible membrane damage are averted. To accomplish such an oxidant flow is equivalent to maintaining the oxygen excess ratio of the cathode at a suitable value. The oxygen excess ratio or oxygen stoichiometry is defined as

$$
\lambda_{O_{2}}=\frac{W_{O_{2}, c a}}{W_{O_{2}, \text { react }}},
$$

where $W_{\mathrm{O}_{2} \text {,react }}$ is the oxygen flow consumed in the reaction and $W_{\mathrm{O}_{2}, \mathrm{ca}}$ is the oxygen partial flow entering the cathode, which depends on the air flow released by the compressor $W_{c p}$, i.e.,

$$
W_{\mathrm{O}_{2}, c a}=\frac{\chi_{\mathrm{O}_{2}} W_{c p}}{1+\omega_{a m b}} .
$$

Here, $\omega_{a m b}$ is the ambient air humidity ratio and $\chi_{O_{2}}$ is the molar fraction of oxygen in the air $\left(\chi_{O_{2}}=0.21\right)$. As $W_{O_{2}, c a}$ is an internal unavailable variable of the system, it is not practical to include it in the control algorithm. This problem was circumvented by inferring information of $W_{\mathrm{O}_{2}, \mathrm{ca}}$ from an accessible variable of the system, such as the air mass flow delivered by the compressor

$W_{c p}=B_{00}+B_{01} \omega_{c p}+B_{02} \omega_{c p}^{2}+\left(B_{10}+B_{01} \omega_{c p}\right) \Psi+B_{02} \Psi^{2}$ being $\Psi=m_{a, \text { hum }} T_{\text {hum }} R_{a} / V_{\text {hum }}+K_{\text {hum }}, \omega_{c p}$ the compressor speed and $m_{a, h u m}$ the humidifier mass of air. The compressor parameters $B_{00}, B_{01}, B_{10}, B_{11}, B_{02}$ and $B_{20}$ can be obtained from [5], $T_{\text {hum }}$ is the humidifier temperature, $V_{\text {hum }}$ is the humidifier volume, $R_{a}$ the air gas constant and $K_{\text {hum }}=P_{\text {sat }}\left(T_{\text {hum }}\right) R H_{\text {hum }}-P_{\text {sat }}\left(T_{a m b}\right) R H_{a m b}$ with $P_{\text {sat }}\left(T_{\text {hum }}\right)$ the vapour saturation pressure at $T_{\text {hum }}, R H_{\text {hum }}$ the relative humidity of the gas at the humidifier output,
$P_{\text {sat }}\left(T_{a m b}\right)$ the vapour saturation pressure at ambient temperature and $\mathrm{RH}_{\text {hum }}$ the relative humidity of ambient air.

Notice that $W_{O_{2}}$,react is directly related to the stack current as follows:

$$
W_{\mathrm{O}_{2}, \text { react }}=G_{\mathrm{O}_{2}} n I_{s t} / 4 \mathrm{~F},
$$

with $G_{\mathrm{O}_{2}}$ the molar mass of oxygen, $n$ the number of cells and Faraday's constant $F$. As presented in the validated model [3], the operating conditions of the system inputs are determined by $V_{c p}$ and $I_{s t}$.

This paper is focused on the oxygen stoichiometry $\lambda_{\mathrm{O}_{2}}$ tracking under continuous changes in the load condition $I_{s t}$, such that

$$
e_{\lambda}=\lambda_{O_{2}}-\lambda_{O_{2}, \text { ref }}
$$

is as small as possible, for both nominal and fault conditions.

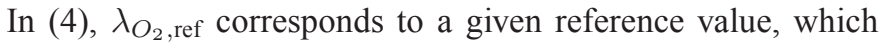
comes from a supervisory controller that considers global objectives related to the efficiency and durability of the overall PEMFC-based system [25].

\section{Unfal Sified CONTROL OF PEM FUEL CELlS}

The UC concept proposed by [18] consists of a set of candidate controllers $\mathbf{K}$ and a switching algorithm that selects the most suitable controller in the set according to a performance criterion based only on experimental input-output data. The main appeal of UC is that there is no need of a plant model to decide if a controller satisfies the performance specifications.

The only a priori information needed about the system is a set of input-output measures $\mathcal{Z}(k)=\{(u(l), y(l)), 0 \leq$ $l \leq k\}$, with $k$ being the discrete time. The performance specifications are stated as a cost-function $\mathcal{V}$ depending on the reference $r$ and on the input $u$ and output $y$. As a consequence, the performance specifications define a subset

$$
\mathcal{T}_{\text {spec }}=\{(r, u, y): \mathcal{V}(r, u, y)<\eta\},
$$

where $\eta$ is a positive scalar bounding the performance specifications. In turn, a candidate controller $K \in \mathbf{K}$ defines also a subset

$$
\mathcal{K}=\{(r, u, y): u=K(r, y)\},
$$

where $K$ must be "causually-left-invertible", i.e., there exists $K^{-1}$ that allows the computation of a fictitious reference $r_{f}$ from $(u, y)$. This reference is the value that $r$ would take if the controller $K$ is inserted in the loop and the input and output of the plant were $(u, y)$. The fictitious reference can be computed from $\mathcal{Z}$ and $K$ without actually inserting the controller in the loop, as follows:

$$
r_{f}=K^{-1}(u, y) .
$$

In this framework, the controller $K$ is said to be unfalsified by the experimental information $\mathcal{Z}$ if

$$
\mathcal{K} \cap \mathcal{Z} \cap \mathcal{T}_{\text {spec }} \neq \emptyset
$$

otherwise the controller is said to be falsified by the measured data. The problem is feasible if the set of candidate controllers includes at least one which stabilizes the system ([19], page 18). 
The selection of the most adequate controller, also denoted the falsification procedure, according to the a posteriori information $(u, y)$ relies on the evaluation of a cost-detectable function. This property guarantees stability and convergence of the adaptive procedure.

The controller set may have a finite or infinite number of controllers. In the first case, all the controllers in the set are tested simultaneously. That could be computationally demanding if it contains a large number of controllers. In the second approach, the set is defined by a control structure that updates its parameters in real time. The selection of the most suitable controller relies on an optimization procedure that computes the best controller parameters. This option could be more computationally efficient but is limited to certain cost functions. Hence, the proper selection of these cost functions is done in such a way that the controller selection results in a convex optimization easy to solve online. The UC technique used here is based on this latter approach.

\section{A. Ellipsoid Unfalsified Control}

The cost function and the controller structure define the falsifier complexity. In particular, the ellipsoid unfalsified control (EUC), by selecting an adequate cost function and a certain control structure, computes the most suitable controller by means of an efficient convex optimization procedure and with proven convergence properties [24]. Most precisely, the controllers are parameterized as

$$
u(k)=\left[\begin{array}{c}
r(k) \\
\Lambda_{u}\left(z^{-1}\right) u(k) \\
y(k) \\
\Lambda_{y}\left(z^{-1}\right) y(k)
\end{array}\right]^{T}\left[\begin{array}{c}
1 / \hat{\theta}_{1} \\
-\hat{\theta}_{2} / \hat{\theta}_{1} \\
-\hat{\theta}_{3} / \hat{\theta}_{1} \\
-\hat{\theta}_{4} / \hat{\theta}_{1}
\end{array}\right],
$$

where $\Lambda_{u}$ and $\Lambda_{y}$ are stable linear filters, $\hat{\theta}_{i}(i=1, \ldots, 4)$ are parameters to be set online, and $z$ is the unity delay. With this parameterization, the fictitious reference can be found as

$$
r_{f}(k)=w^{T}(u, y, k) \theta
$$

where

$$
w=\left[\begin{array}{c}
u(k) \\
\Lambda_{u}\left(z^{-1}\right) u(k) \\
y(k) \\
\Lambda_{y}\left(z^{-1}\right) y(k)
\end{array}\right], \quad \theta=\left[\begin{array}{c}
\theta_{1} \\
\theta_{2} \\
\theta_{3} \\
\theta_{4}
\end{array}\right] .
$$

The controller parameterization and the computation of the fictitious references are illustrated in Fig. 2. Notice the difference between the parameter $\hat{\theta}$ of the current controller and $\theta$ the parameter under performance evaluation by the UC algorithm.

The performance criterion is cast in the form of model reference tracking as

$$
\left|e_{f}(\theta, k)\right|+\kappa|u(k)| \leq \Delta(k),
$$

where $e_{f}(k)=G_{m}\left(z^{-1}\right) r_{f}(\theta, k)-y(k), G_{m}$ is a stable system that defines the desired behavior and $\Delta(\cdot)$ is a time dependent bound. Then, the set of controller parameters that satisfy the performance specifications is given by

$$
\mathcal{U}(k)=\left\{\theta:-\hat{\Delta}(k) \leq e_{f}(\theta, k) \leq \hat{\Delta}(k)\right\},
$$

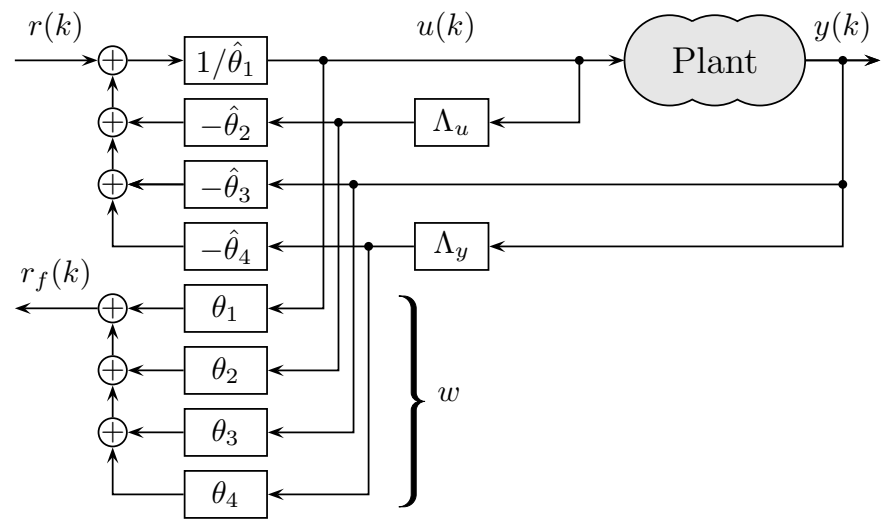

Fig. 2. Controller parameterization and fictitious reference computation

where $\hat{\Delta}(k)=\Delta(k)-\kappa|u(k)|$. The set of controllers is given by (7) and the parameter set

$$
\mathcal{E}(k)=\left\{\theta:\left(\theta(k)-\theta_{c}(k)\right)^{T} \Sigma(k)\left(\theta(k)-\theta_{c}(k)\right)\right\},
$$

where $\mathcal{E}(k)$ is an ellipsoid of center $\theta_{c}(k)$ and size defined by the positive definite matrix $\Sigma(k)$ [24].

With these definitions, the controller and specification sets are parameterized in $\theta$ and condition (6) results in

$$
\mathcal{E}(k) \bigcap \mathcal{U}(k) \neq \emptyset \text {. }
$$

That is, the set of unfalsified controllers is given by the parameters $\theta$ in the intersection of $\mathcal{E}(k)$ and $\mathcal{U}(k)$. Therefore, the falsification algorithm reduces to shrinking the ellipsoid volume $(\operatorname{vol}(\mathcal{E}(k)))$ by changing the matrix $\Sigma(k)$, to check the intersection of $\mathcal{E}(k)$ and $\mathcal{U}(k)$ and to select a new $\hat{\theta} \in\{\mathcal{E}(k) \bigcap \mathcal{U}(k)\}$.

The original EUC algorithm was intended for time-invariant systems and the volume of the ellipsoid was reduced as long as the a posteriori information increased and thus the controller parameters converged to the controller that satisfied the performance specifications. In other words, when the number of samples of $(u, y)$ increases, the information is used to remove those controllers that do not satisfy the performance criterion. In case of time varying or nonlinear systems, a controller falsified for certain operating conditions could satisfy the performance criterion in other operating points. Therefore, the EUC algorithm needs some modification in order to cover these cases. Here, the expansion of the ellipsoidal volume when no controller is falsified, is proposed. More precisely, if the current controller parameter are not falsified after $k_{t h}$ samples, the ellipsoid volume $\operatorname{vol}(\mathcal{E}(k))$ is expanded by changing the matrix $\Sigma$ as follows

$$
\Sigma(k+1)=\Sigma(k) \beta^{p},
$$

where $\beta>1$ and $p$ increase by 1 each time the current controller remains unfalsified during more than $k_{t h}$ samples. The expansion continues until the controller is falsified or the initial volume is reached.

\section{B. EUC for PEMFC}

To design an EUC control algorithm it is necessary to choose the filters $\Lambda_{u}$ and $\Lambda_{y}$ which define the controller set, 
and the transfer function $G_{m}$ to define the desired behavior. Although EUC does not require a priori information of the plant, it is always useful to have a rough idea about its dynamics and the structure needed to achieve the desired closed-loop behavior.

In the case of the PEMFC, the system behavior around an operating point can be roughly approximated by a second order system of the form

$$
G(z)=\frac{\lambda_{O_{2}}(z)}{V_{c p}(z)}=K_{f c} \frac{z-a}{(z-b)(z-c)} .
$$

By selecting

$$
\Lambda_{u}(z)=\Lambda_{y}(z)=\frac{K_{\Lambda}}{z-q}
$$

and the control law

$u(k)=\frac{1}{\theta_{1}} r(k)-\frac{\theta_{2}}{\theta_{1}} \cdot \frac{K_{\Lambda}}{z-q} u(k)-\left(\frac{\theta_{3}}{\theta_{1}}+\frac{\theta_{4}}{\theta_{1}} \cdot \frac{K_{\Lambda}}{z-q}\right) y(k)$,

and for the particular values $\theta_{3}=1$ and $\theta_{4}=0$, the controller results

$$
u(k)=\frac{1}{\theta_{1}} \cdot \frac{z-q}{z-\left(q-\theta_{2} K_{\Lambda} / \theta_{1}\right)}(r(k)-y(k)) .
$$

With proper values of $\theta_{1}$ and $\theta_{2}$, it is possible to obtain a closed-loop transfer function of the form

$$
G_{c l}(z)=\frac{\lambda_{O_{2}}(z)}{\lambda_{O_{2}, \mathrm{ref}}(z)}=\frac{K_{c l}}{z-q_{c l}}
$$

Therefore, it is reasonable that the desired closed-loop behavior given by $G_{m}$ has the form of $G_{c l}$ in (17).

\section{EXPERIMENTAL RESUlts}

This section describes the different scenarios considered for testing the effectiveness of the proposed control approach. For every scenario, the main results are discussed through the most relevant variables involved in each case. They include typical performance tests and the effect of faults in different parts of the PEMFC-based system. Before analyzing the experimental results, a brief description of the experimental test bench and the particular EUC settings are presented.

\section{A. Workplace Setup}

The control strategy was implemented in a complete data acquisition and control system. It is composed of two computers (each with four i5 core processors at $2.6 \mathrm{GHz}$ clock frequency): the host and the real-time operating system (RTOS). The former provides the software development environment and the graphical user interface. It is responsible for the start up, shut down, configuration changes and control settings during operation. The latter implements the control algorithms and the data acquisition via a field-programmable gate array (FPGA), in order to have high speed data processing. Control, security and monitoring tasks are conducted by a CompactRIO (reconfigurable Input/Output) system from National Instruments ${ }^{\mathrm{TM}}$. In order to record the analog sensor signals, a 32-channel 16-bit analog input module from National Instruments ${ }^{\mathrm{TM}}$ is used (NI-9205). An 8-channel, digital

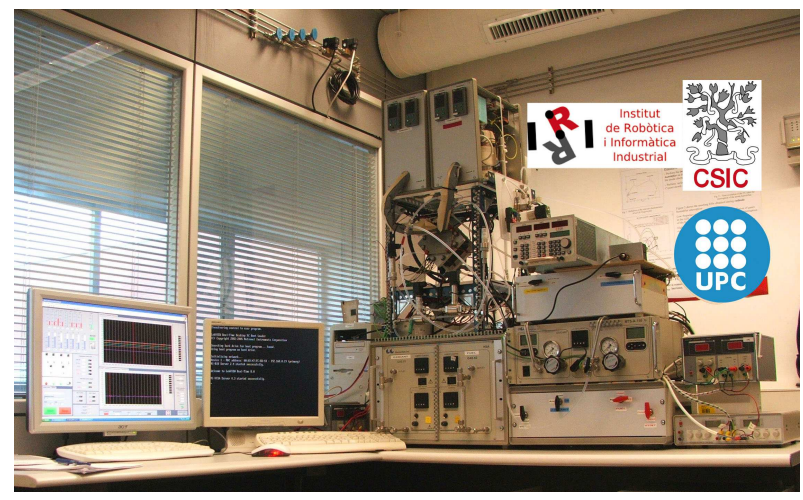

Fig. 3. Picture of the laboratory test station at IRI (CSIC-UPC)

input/output (I/O) module generates the necessary transistortransistor logic (TTL) signals for different security and diagnostic tools. Fig. 3 shows the laboratory setup used in the experiments.

\section{B. EUC Controller Setup}

The EUC algorithm has been developed in Matlab ${ }^{\circledR}$ and then cross-compiled into a LabView ${ }^{\circledR}$ environment by means of a $D L L$ file obtained through the Matlab ${ }^{\circledR}$ Real-Time Workshop Toolbox.

The tracking error was bounded with the function

$$
\Delta(k)=0.25+1.9 \mathrm{e}^{-0.02 k},
$$

which ensures a $2 \%$ tracking error and relaxes the error during the initial transients, avoiding excessive controller falsifications.

The filters were selected as

$$
\Delta_{y}(z)=\Delta_{u}(z)=\frac{0.00897}{z-0.991}
$$

and the reference model as

$$
G_{m}(z)=\frac{0.0198}{z-0.9802}
$$

These transfer functions were selected based on linear models identified at several operating points, therefore the adopted control structure allows achieving the desired closed-loop behavior. The sampling time was $0.01 \mathrm{~s}$.

The initial value of the controller parameters was

$$
\theta_{0}=\left[\begin{array}{llll}
2 & -1.99 & 1 & 0
\end{array}\right]^{T} .
$$

The parameters for the expansion of the ellipsoid volume were set as $\beta=1.5$ and $k_{t h}=100$.

\section{Complete Control Strategy}

The UC is complemented with a bumpless and a flow control to help in the start-up of the system. This complementary start-up controller acts as a safety strategy to avoid undesired consequences in the fuel cell stack durability, regulating the air mass inflow from the compressor. Thus, $W_{c p}$ is regulated towards a convenient value in such a way that $\lambda_{O_{2}}$ reaches values close to its desired reference $\lambda_{O_{2}}$,ref (given that both 


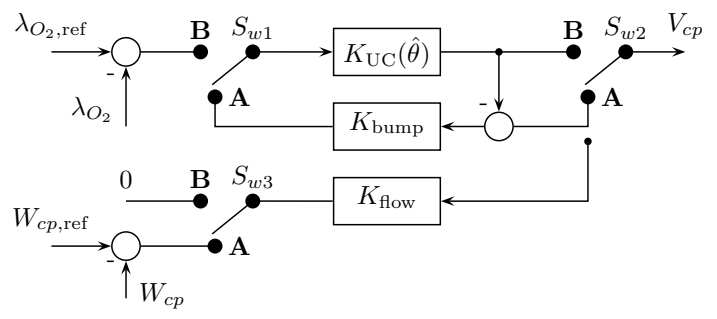

Fig. 4. Complete control scheme including the UC and the start-up controllers

variables $W_{c p}$ and $\lambda_{O_{2}}$ are related by means of (1) and (2)). Therefore, a smooth starting behavior of $\lambda_{O_{2}}$ is achieved. The complete control scheme is sketched in Fig. 4.

In this initial stage, the switches $S_{w 1}=S_{w 2}=S_{w 3}$ are set at position $\mathbf{A}$ and the controller

$$
K_{\text {flow }}(z)=0.43+\frac{0.043}{z-1}
$$

tracks a predefined profile leading the system to a suitable flow condition before starting the stoichiometry control. This PI controller was designed experimentally based on the step response of the system under the initial operating conditions to ensure a settling time lower than $1 \mathrm{~s}$.

The bumpless controller

$$
K_{\text {bump }}(z)=0.3175+\frac{0.2}{z-1}
$$

ensures a smooth transition from flow to stoichiometry control. This PI controller was designed to ensure that $K_{\mathrm{UC}}\left(\theta_{0}\right)$ achieves a rapid tracking of the signal $V_{c p}$ produced by $K_{\text {flow }}$.

Once a pre-set time is reached, the $S_{w 1}=S_{w 2}=S_{w 3}$ are set at position $\mathbf{B}$ and the control switches to stoichiometry control. Initially, the EUC starts with a fixed initial control given by $\theta_{0}$. This can be a conservative controller that covers the complete operating envelope in a stable way, but with poor performance. Once the EUC is fully operative, the switching algorithm is responsible for finding a more suitable parameter $\theta$ to achieve a better performance in the actual operating conditions.

\section{Experimental Scenarios}

In order to evaluate the performance of the proposed closedloop control scheme, the following realistic scenarios are considered for covering not only nominal (faultless) situations but also the effect of real faults in the system. Note that these tests include a real set of safety measures and devices that avoid any hazardous behavior of the test bench (like over pressures, temperatures or currents). The anode line is also monitored by a higher level supervisor, avoiding any irreversible damages in the cells due to high differential pressure between anode and cathode.

1) Scenario 1. Start-up Controller and Reference Tracking: this scenario considers two parts; the system behaviour with a start-up flow controller and the reference tracking performance. First of all, in order to carry the system variables towards an initial operation regime, the overall control structure considers the initial regulation of the compressor flow $W_{c p}$ at
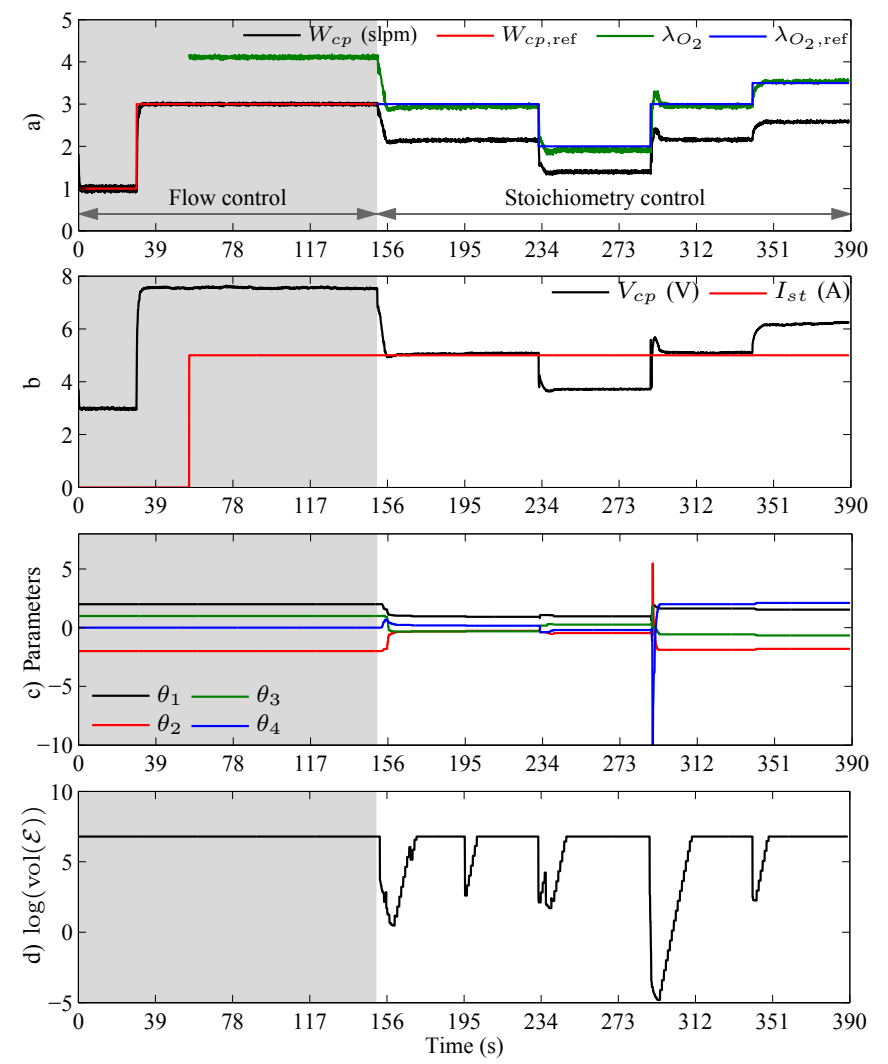

Fig. 5. Start-up and closed-loop response for several step changes in the stoichiometry reference (Scenario 1)

a given value, close enough to nominal operating points when the stack is delivering electrical power. In this initial stage, the falsification algorithm is out of the loop and will only be activated after $W_{c p}$ reaches its reference value

$$
W_{c p, \text { ref }}=\left(1+\omega_{a t m}\right) \lambda_{o 2, r e f} G_{O_{2}} n I_{s t} /\left(4 F \chi_{o 2}\right) .
$$

Fig. 5 presents the behaviour of the system variables for this scenario. Notice that the stoichiometry is not well defined until $I_{s t}$ is greater than zero.

After $150 \mathrm{~s}$ of flow regulation, the closed-loop system switches to an initial stabilizing controller $\left(\theta=\theta_{0}\right)$ before activating the EUC controller at time $t=152 \mathrm{~s}$ (see the transitions in the ellipsoid volume parameter $\operatorname{vol}(\mathcal{E}(k))$ in Fig. 5d) and considers different values for the oxygen stoichiometry reference $\lambda_{O_{2}}$, ref ranging from 2 to 3.5 . Here, the stack current $I_{s t}$ remains constant at $5 \mathrm{~A}$. This is a typical scenario where the oxygen stoichiometry of a PEMFC-based system is changed to obtain different net powers. Although the flow control is no longer connected, $W_{c p}$ follows the stoichiometry evolution due to their relation given by (1) and (2). Once the EUC is activated, notice the suitable change of parameters $\theta_{i}$ in Fig. $5 \mathrm{c}$, which induces smooth changes in the control signal $V_{c p}$ (Fig. $5 \mathrm{~b}$ ) in order to adapt the controller to different operating conditions associated with the different

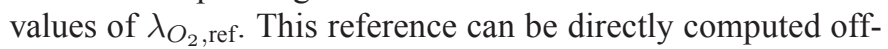
line or through an extremum seeking algorithm like the one presented in [25], where the goal is to optimize the overall system efficiency. The bottom plot shows the evolution of the 

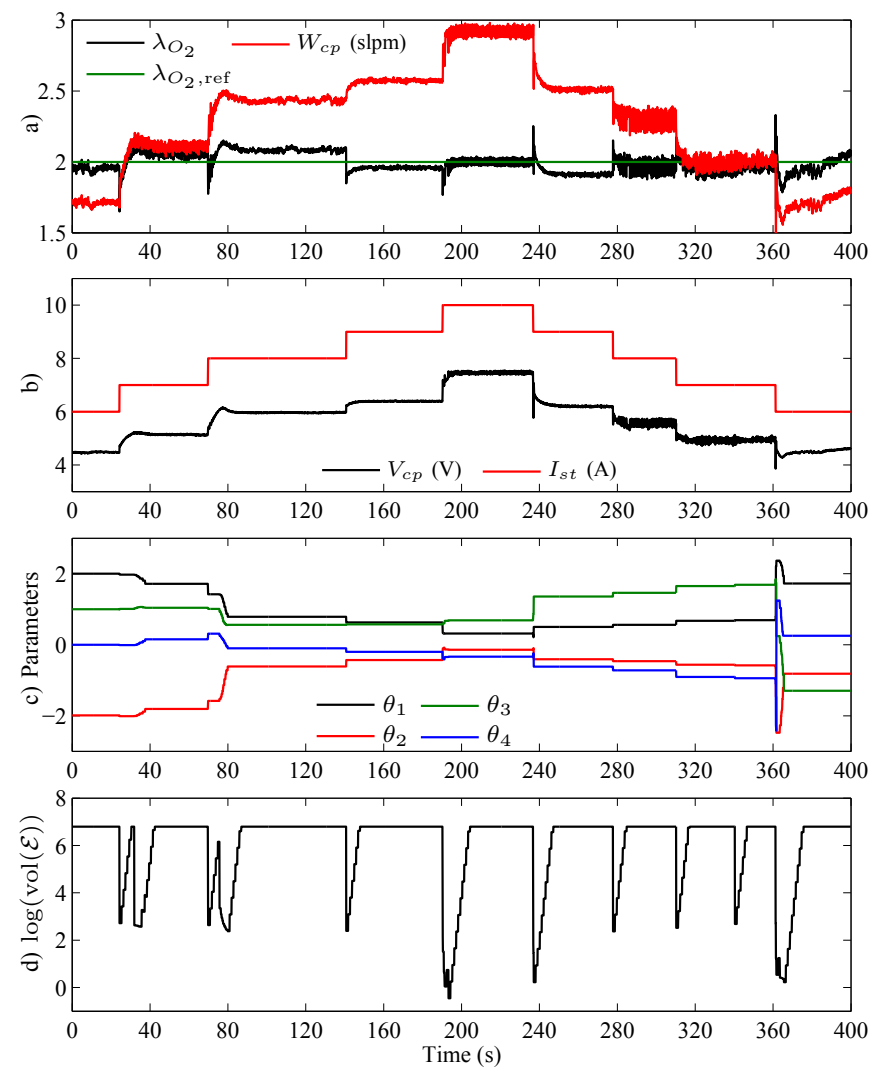

Fig. 6. Closed-loop response for several step changes in $I_{s t}$ (Scenario 2)

ellipsoid volume $\operatorname{vol}(\mathcal{E}(k)$ ) (in logarithmic scale). It can be seen that after $k_{t h}=100$ samples without any controller falsification, the algorithm expands the volume to be better prepared for new operating conditions.

2) Scenario 2. Disturbance Rejection: considering that the desired value of $\lambda_{\mathrm{O}_{2} \text {,ref }}$ is already reached, it is also important to evaluate the performance of the EUC-based closed-loop system when changes in the load current $I_{s t}$ take place. To reproduce this typical working case, $\lambda_{\mathrm{O}_{2}}$, ref was set at 2 , while different values of $I_{s t}$ have been required from the PEMFC stack. Fig. 6 shows the main variables related to this test. Notice that $\lambda_{O_{2}}$ is rapidly reaching the new steadystate desired value after each change of $I_{s t}$. Meanwhile, the parameters $\theta_{i}$ are being adapted to this end (Fig. 6c), with changes in $I_{s t}$ between 6 and $10 \mathrm{~A}$ and smooth changes of the control signal $V_{c p}$. The lower plots shows the updates of the controller parameters and the changes in the ellipsoid volume $\operatorname{vol}(\mathcal{E}(k))$ when the operating conditions change as a consequence of changes in $I_{s t}$. It can be seen that parameters $\theta_{i}$ change more than once for constant values of $I_{s t}$. This is mainly a consequence of the noise in the measures of $V_{c p}$.

Besides, Fig. 7 shows the stoichiometry regulation under a demanding scenario, in which the current $I_{s t}$ increases and decreases in step changes of 6 A. Even under demanding conditions, the proposed EUC control scheme is capable of rapidly returning the stoichiometry to the set-point value. To properly handle these abrupt step changes, faster devices such as supercapacitors and/or batteries should be connected in parallel with the PEMFC system.
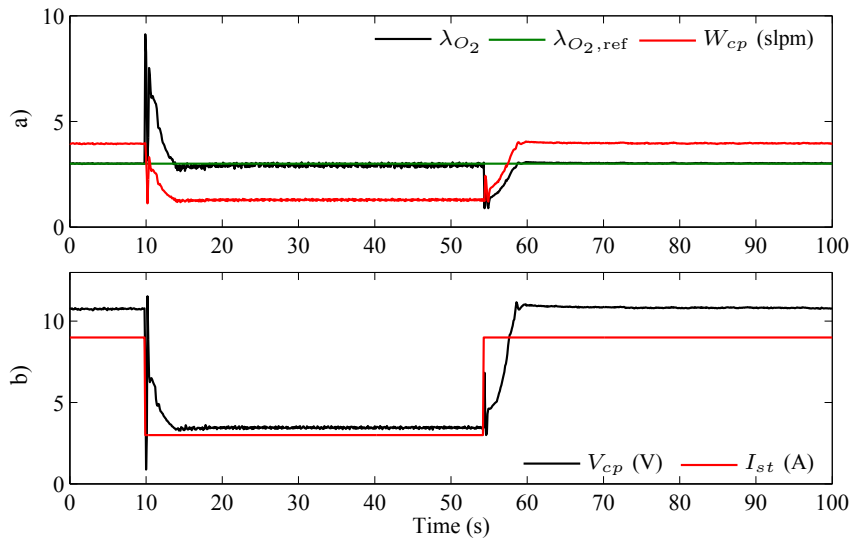

Fig. 7. Closed-loop response for $6 \mathrm{~A}$ step changes in $I_{s t}$ (Scenario 2)

3) Scenario 3. Cathode Outflow Fault: this scenario considers the effect of a couple of faults in the performance of the PEMFC-based system. Faults in this case are related to the cathode outflow in the following way: (i) there is a flow blockage (FB) that causes the increase of the cathode inlet pressure $P_{c a}$, and (ii) there is a flow leak (FL) that is compensated by increasing $V_{c p}$ without affecting $P_{c a}$. The goal is to check the behavior of the EUC-based closed loop when rejecting these changes in the cathode line, while both $I_{s t}$ and $\lambda_{\mathrm{O}_{2}}$ remain constant at $5 \mathrm{~A}$ and 3 , respectively, along the whole experiment. Fig. 8 shows the system variables related to this test. The magnitude of the FB fault can be quantified by either analyzing the behavior of $P_{c a}$ (Fig. 8b), or computing the compressor power by means of $V_{c p}$ and $I_{c p}$, both plotted in the same figure. Since the FB fault appearing at $t=35 \mathrm{~s}$ progressively increases $P_{c a}$, the EUC controller suitably adapts the parameters during that effect (see transitions of $\operatorname{vol}(\mathcal{E}(k)$ ) after $35 \mathrm{~s}$ ). In the case of the FL fault, its magnitude can be quantified by observing $V_{c p}$ since $P_{c a}$ is not affected due to the compensation performed by the manipulated input after $t=255 \mathrm{~s}$. It should be noticed that the proposed control scheme is capable to properly reject the effect of the considered faults and, after a slight deviation, $\lambda_{\mathrm{O}_{2}}$ returns to its desired reference value. The controller also allows to recover the system even when the fault disappears and the nominal behavior is recovered.

As an additional evaluation of the proposed control scheme, Fig. 9 shows the disturbance rejection capability under a flow-blockage fault. The EUC controller reaches the proper recovery of $\lambda_{\mathrm{O}_{2}}$ when several changes of $I_{s t}$ were performed.

4) Scenario 4. Compressor Fault: Here, a different fault is considered, which is related to the capacity of the air supply from the compressor connected to the PEMFC cathode. The fault affects the compressor by changing the inertia and nominal friction of its motor shaft. Again, the goal is to check the behavior of the EUC-based closed loop when rejecting this fault while both $I_{s t}$ and $\lambda_{\mathrm{O}_{2}}$ remain constant at $6 \mathrm{~A}$ and 2, respectively. Fig. 10 shows the system variables during this test. The magnitude of the fault in this case is strongly related to $I_{c p}$ but notice that for this case, $P_{c a}$ remains constant (Fig. 10b). The fault appears at time $t=50 \mathrm{~s}$, disappears at 

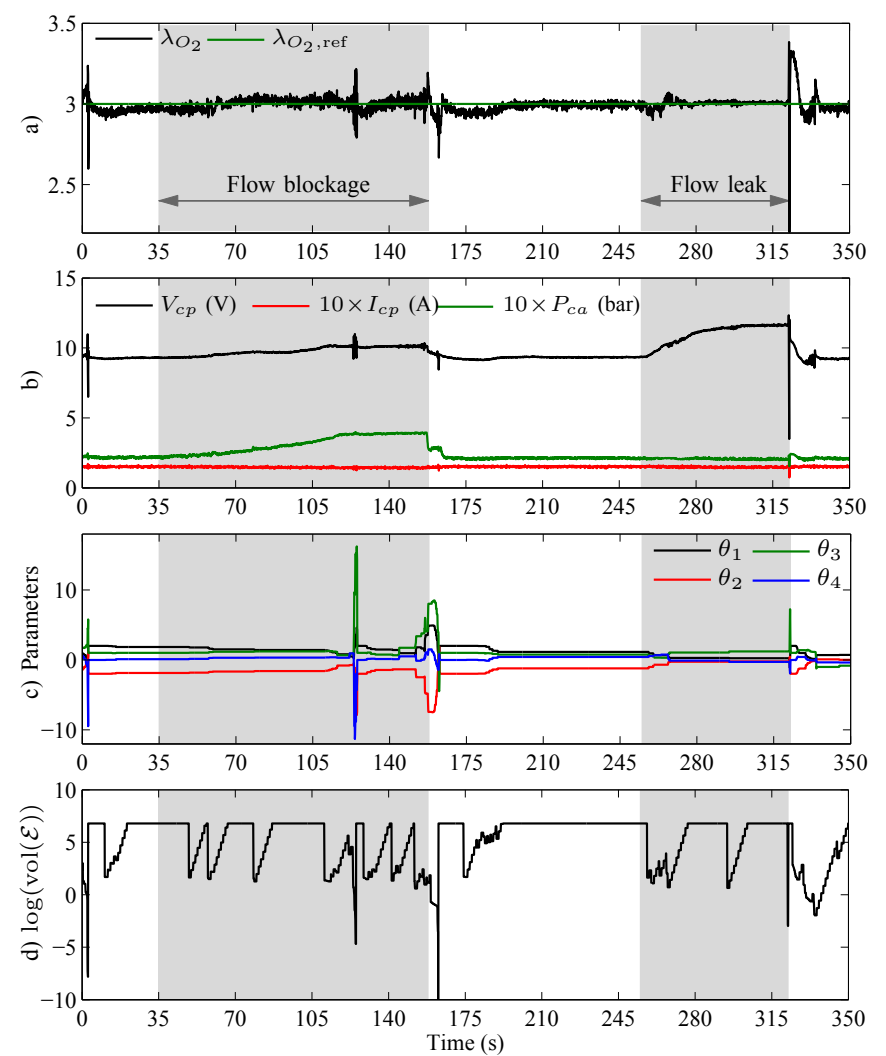

Fig. 8. Closed-loop response during flow-blockage and flow-leak faults (Scenario 3)
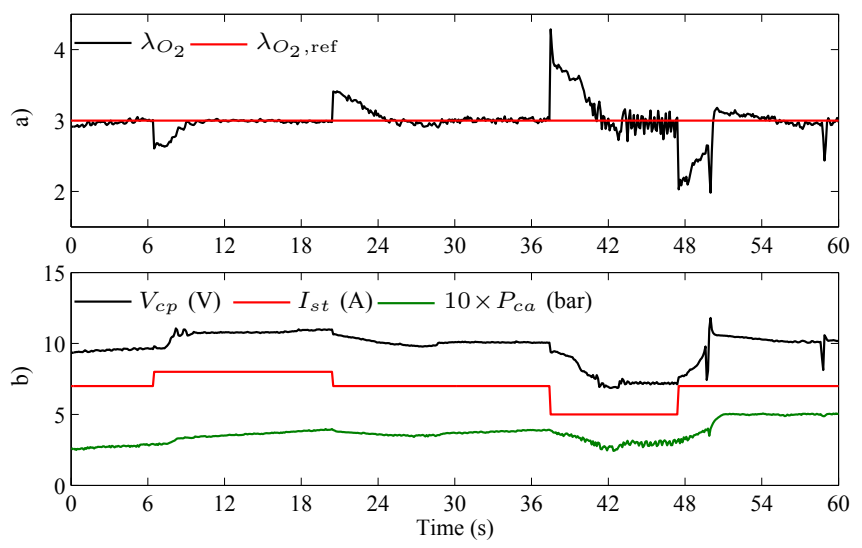

Fig. 9. Closed-loop response for $I_{s t}$ changes under a flow-blockage fault (Scenario 3)

$t=106 \mathrm{~s}$ and appears again at $t=146 \mathrm{~s}$. The EUC controller adapts the parameters $\theta_{i}$ conveniently while reducing the stoichiometry regulation error as much as possible.

The results presented in this paper mainly highlight the implicit fault tolerance capabilities given by the EUC scheme (due to its data-driven control nature) independently of knowing the particular way the faults affect the system. As stated in the Introduction, several authors have reported the design and implementation of FTC techniques for PEMFC systems, which explicitly use the system model [15], [16] unlike the fault tolerance capabilities of the proposed model-free approach. On the other hand, reported adaptive schemes for
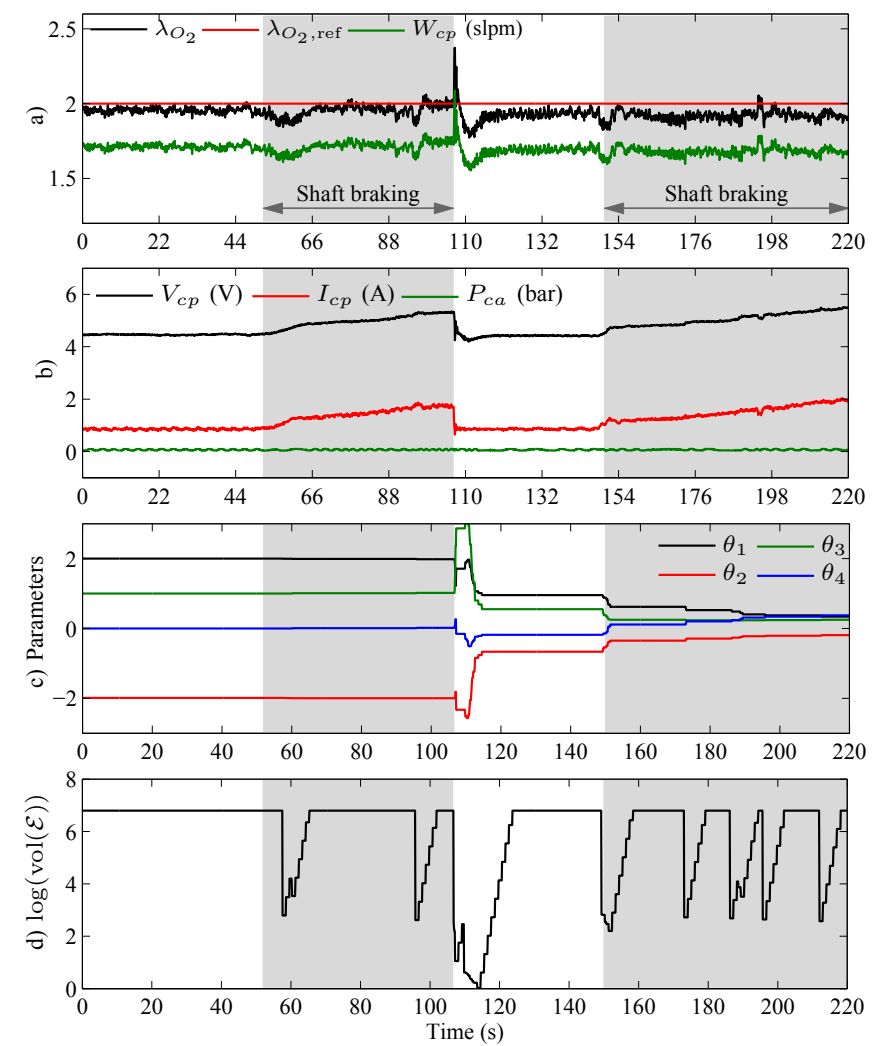

Fig. 10. Closed-loop response during a compressor fault (Scenario 4)

PEMFCs address the manipulation of the air mass flow for controlling $\lambda_{O_{2}}$ [27], [8] and the online system identification and efficiency management by controlling $\lambda_{\mathrm{O}_{2}}$, relative humidity and stack temperature [9]. Although those approaches show experimental evidences of their proper operation under nominal conditions, they do not consider the effects of faults over the performance of the closed loop.

\section{CONCLUSions}

A fault-tolerant control for PEMFC was proposed and experimentally tested in a laboratory test bench. The proposed control is based on EUC that allows adapting the controller parameters by evaluating the closed-loop performance solely from measures of the compressor voltage (control input) and the oxygen stoichiometry (controlled output). The EUC algorithm does not rely on a plant model, which makes it suitable for dealing with complex systems and also to tackle faults in the cathode outflow or in the compressor. Four experimental scenarios have shown that the proposed UC control is capable of effectively working in different operating conditions and most common faults in PEMFCs. A start-up mass-flow control strategy has also been introduced, which avoids abrupt changes in the system variables when the initial conditions are far away from the nominal values.

\section{ACKNOWLEDGEMENTS}

All the experimental tests were performed at the PEM Fuel Cells Laboratory of the Institut de Robòtica i Informàtica Industrial (CSIC-UPC, Barcelona, Spain) and only possible due to its advanced equipment and proficient technical staff. 


\section{REFERENCES}

[1] "U.S. Department of Energy. Hydrogen, Fuel Cells And Infrastructure Technologies Program: Multi-Year Research, Development And Demonstration Plan: planned program activities for 2005-2015. DOE Website. Section 3.4 Fuel Cells." Tech. Rep., 2005.

[2] J. Blanchard, "Smart energy solutions using fuel cells," in Proc. of the 33rd INTELEC, Amsterdam, NL, 2011, pp. 1-8.

[3] C. Kunusch, P. Puleston, M. Mayosky, and A. Husar, "Control oriented modelling and experimental validation of a PEMFC generation system," IEEE Trans. Energy Convers., vol. 6, no. 3, pp. 851-861, 2011.

[4] C. Ziogou, S. Papadopoulou, M. C. Georgiadis, and S. Voutetakis, "Online nonlinear model predictive control of a PEM fuel cellsystem," $J$. Process Control, vol. 23, no. 4, pp. 483 - 492, 2013.

[5] C. Kunusch, P. Puleston, M. Mayosky, and L. Fridman, "Experimental results applying second order sliding mode control to a PEM fuel cell based system," Control Eng. Pract., vol. 21, no. 5, pp. 719 - 726, 2013.

[6] J. Pukrushpan, A. Stefanopoulou, and H. Peng, Control of Fuel Cell Power Systems. London, UK: Springer London Ltd, 2004.

[7] A. Niknezhadi, M. Allué-Fantova, C. Kunusch, and C. OcampoMartinez, "Design and implementation of $\mathrm{LQR} / \mathrm{LQG}$ strategies for oxygen stoichiometry control in PEM fuel cells based systems," J. Power Sources, vol. 196, no. 9, pp. 4277-4282, 2011.

[8] F. Bianchi, C. Kunusch, C. Ocampo-Martinez, and R.S. Sánchez-Peña, "A gain-scheduled LPV control for oxygen stoichiometry regulation in PEM fuel cell systems," IEEE Trans. Control Syst. Technol., vol. 22, no. 5, pp. 1837-1844, 2014

[9] S. Kelouwani, K. Adegnon, K. Agbossou, and Y. Dube, "Online system identification and adaptive control for PEM fuel cell maximum efficiency tracking," IEEE Trans. Energy Convers., vol. 27, no. 3, pp. 580-592, 2012.

[10] M. Blanke, M. Kinnaert, J. Lunze, and M. Staroswiecki, Diagnosis and Fault-Tolerant Control. Heidelberg, Germany: Springer-Verlag, 2006.

[11] A. Rosich, R. Sarrate, and F. Nejjari, "On-line model-based fault detection and isolation for PEM fuel cell stack systems," Appl. Math. Model., vol. 38, no. 11-12, p. 2744 2757, 2014.

[12] N. Lowery, M. Vahdati, R. Potthast, and W. Holderbaum, "Classification and fault detection methods for fuel cell monitoring and quality control," Journal of Fuel Cell Science Technology, vol. 10, no. 2, pp. 1-8, 2013.

[13] A. Hernandez, D. Hissel, and R. Outbib, "Modeling and fault diagnosis of a polymer electrolyte fuel cell using electrical equivalent analysis," IEEE Trans. Energy Convers., vol. 25, no. 1, pp. 148-160, 2010.

[14] W. Yang, K. Lee, S. Junker, and H. Ghezel-Ayagh, "Fuzzy fault diagnosis and accommodation system for hybrid fuel-cell/gas-turbine power plant," IEEE Trans. Energy Convers., vol. 25, no. 4, pp. 1187 1194, 2010

[15] L. Xu, J. Li, M. Ouyang, J. Hua, and X. Li, "Active fault tolerance control system of fuel cell hybrid city bus," Int. J. Hydrogen Energy, vol. 35 , no. 22 , pp. $12510-12520,2010$.

[16] D. Feroldi, "Fault diagnosis and fault tolerant control of PEM fuel cell systems," in PEM Fuel Cells with Bio-Ethanol Processor Systems. Springer Verlag, 2012, pp. $185-206$

[17] I. Markovsky, "Closed-loop data-driven simulation," Int. J. Control, vol. 83, no. 10, pp. $2134-2139,2010$.

[18] M. Safonov and T. Tsao, "The unfalsified control concept and learning," IEEE Trans. Autom. Control, vol. 42, no. 6, pp. 843 - 847, 1997.

[19] M. Stefanovic and M. Safonov, Safe Adaptive Control. London, UK: Springer Verlag, 2011

[20] E. Nabati and S. Engell, "Data-driven adaptive control: Making unfalsified control work better," in Proc. of the 18th IFAC World Congress, Milano, Italy, 2011, pp. $1285-1290$.

[21] A. Neamtu and A. Stoica, "Unfalsified Control: Application to automatic flight control system design," NCAS Bulletin, vol. 3, no. 3, pp. 103 114, 2011.

[22] A. Wolniakowski and A. Mystkowski, "Application of unfalsified control theory in controlling MAV," Solid State Phenom., vol. 198, pp. 171-175, 2013.

[23] J. van Helvoort, B. de Jager, and M. Steinbuch, "Unfalsified control using an ellipsoidal unfalsified region applied to a motion system," in Proc. of the 16th IFAC World Congress, Prague, Czech, 2005, pp. 1-6.

[24] _ "Direct data-driven recursive controller unfalsification with analytic update," Automatica, vol. 43, no. 12, pp. 2034 - 2046, 2007.

[25] C. Kunusch and F. Castaños, "Extremum seeking algorithms for minimal hydrogen consumption in pem fuel cells," in Proc. of the American Control Conference, Washington DC, 2013, pp. 1144-1149.
[26] C. Ocampo-Martinez, R.S. Sánchez-Peña, F. Bianchi, and A. Ingimundarson, "Integrating unfalsified control and fault diagnosis for fault-tolerant control," Int. J. Syst. Sci., 2014, (submitted).

[27] J. Zhang, G. Liu, W. Yu, and M. Ouyang, "Adaptive control of the airflow of a PEM fuel cell system," J. Power Sources, vol. 179, no. 2, pp. $649-659,2008$.

F.D. Bianchi received the B.S. and Ph.D. degrees in electronic engineering from the National University of La Plata (UNLP), Argentina, in 1999 and in 2005, respectively. From 1999 to 2006, he was a Ph.D. student and a Postdoctoral Fellow at the Laboratory of Industrial Electronic, Control and Instrumentation (LEICI, UNLP, La Plata, Argentina). From 2006 to 2010, he was a Postdoctoral Researcher at the Technical University of Catalonia, Barcelona, Spain. In 2010, he joined the Power Electronics and Electric Power Grids Group, Catalonia Institute for Energy Research (IREC), Barcelona, as a Scientific Researcher. His main research interests include robust control and linear parameter-varying systems and their applications to the control of renewable energy conversion systems.

C. Ocampo-Martinez (StM'97-M'12-SM'13) received his Electronic Engineering degree and his MSc degree in Industrial Automation from the National University of Colombia, Campus Manizales, in 2001 and 2003, respectively. In 2007, he received his PhD Degree in Control Engineering from the Technical University of Catalonia (Barcelona, Spain). After a year as postdoctoral fellow of the ARC Centre of Complex Dynamic Systems and Control (University of Newcastle, Australia), he was with the Spanish National Research Council (CSIC) at the Institut de Robòtica i Informàtica Industrial (IRI) in Barcelona as a Juan de la Cierva research fellow. Since 2011, he is Assistant Professor at the Technical University of Catalunya, Automatic Control Department (ESAII). His main research interests are in the areas of constrained model predictive control, large-scale systems management, nonlinear dynamics and industrial applications.

C. Kunusch (StM'03-M'10) received his B.S., MSc and Ph.D. degrees in electronic engineering from the National University of La Plata (UNLP), Argentina, in 2003, 2006 and in 2009, respectively. In 2010, he was appointed as Postdoctoral Fellow of the Spanish National Research Council (CSIC) at the Institut de Robòtica i Informàtica Industrial (IRI) in Barcelona (Spain). Since 2014, he joined the Electric Drives Pre-Development Team of Brose Fahrzeugteile, Würzburg (Germany), as Senior Researcher. His main research interests include variable structure systems and their applications to the control and observation of fuel cell based systems and electric drives.

R.S. Sánchez-Peña (StM'86-M'88-SM'00) received the Electronic Engineer degree from the University of Buenos Aires (UBA, 1978), and the M.Sc. and Ph.D. from the California Institute of Technology (1986, 1988), both in Electrical Engineering. In Argentina he worked in CITEFA, CNEA and the space agencies CNIE and CONAE. He collaborated with NASA in aeronautical and satellite projects and with the German (DLR) and Brazilian (CTA/INPE) space agencies. He was Full Professor at UBA (1989-2004), ICREA Senior Researcher at the Universitat Politenica de Catalunya (20052009, Barcelona) and visiting Prof./Researcher at several Universities in the USA and the EU. He has consulted for ZonaTech (USA), STI and VENG (Argentina) in aerospace applications and with Alstom-Ecotecnia (Spain) in wind turbine applications. He is recipient of the Premio Consagración in Engineering by the National Academy of Exact, Physical and Natural Sciences (ANCEFN, Argentina) and the Group Achievement Award from NASA as a Review Board member for the Aquarius/SAC-D satellite. Since 2009 he is Director of the PhD Department in Engineering at the Buenos Aires Institute of Technology (ITBA) and a CONICET Principal Investigator. He has applied Identification and Control techniques to acoustical, mechanical and aero \& astronautical engineering and also to type I Diabetes. 